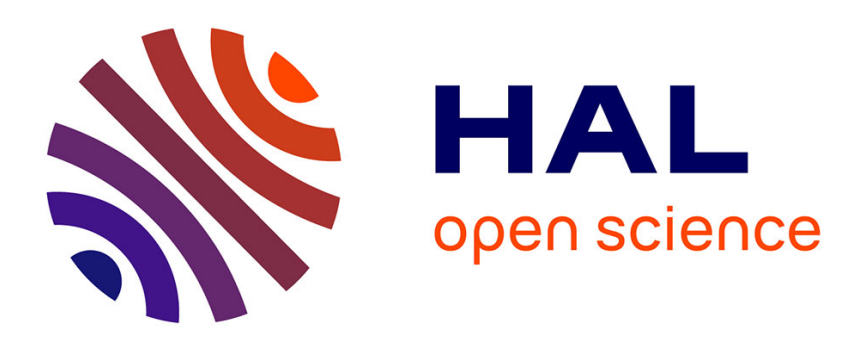

\title{
Formal Verification of User-Level Real-Time Property Patterns
}

\author{
Ning Ge, Marc Pantel, Silvano Dal Zilio
}

\section{To cite this version:}

Ning Ge, Marc Pantel, Silvano Dal Zilio. Formal Verification of User-Level Real-Time Property Patterns. 11th International Symposium on Theoretical Aspects of Software Engineering (TASE 2017), Sep 2017, Sophia Antipolis, France. 8p. hal-01589479

\section{HAL Id: hal-01589479 \\ https://hal.science/hal-01589479}

Submitted on 18 Sep 2017

HAL is a multi-disciplinary open access archive for the deposit and dissemination of scientific research documents, whether they are published or not. The documents may come from teaching and research institutions in France or abroad, or from public or private research centers.
L'archive ouverte pluridisciplinaire HAL, est destinée au dépôt et à la diffusion de documents scientifiques de niveau recherche, publiés ou non, émanant des établissements d'enseignement et de recherche français ou étrangers, des laboratoires publics ou privés. 


\section{Formal Verification of User-Level Real-Time Property Patterns}

\author{
Ning Ge \\ School of Software, Beihang University \\ Beijing, China \\ gening@buaa.edu.cn
}

\author{
Marc Pantel \\ IRIT/INPT \\ Toulouse, France \\ marc.pantel@enseeiht.fr
}

\author{
Silvano Dal Zilio \\ LAAS-CNRS \\ Toulouse, France \\ dalzilio@laas.fr
}

\begin{abstract}
To ease the expression of real-time requirements, Dwyer, and then Konrad, studied a large collection of existing systems in order to identify a set of real-time property patterns covering most of the useful use cases. The goal was to provide a set of reusable patterns that system designers can instantiate to express requirements instead of using complex temporal logic formulas. A limitation of this approach is that the choice of patterns is more oriented towards expressiveness than efficiency; meaning that it does not take into account the computational complexity of checking patterns. For this purpose, we define a set of verification-dedicated, atomic property patterns for qualitative and quantitative real-time requirements. End-user requirements can then be expressed as a composition of these patterns using a predefined meta-model and a mapping library. These properties can be checked efficiently using a set of elementary observers and a model checking approach.
\end{abstract}

Index Terms-real-time requirements, property pattern, observer, model checking, Time Petri net

\section{INTRODUCTION AND RELATED WORK}

Real-time requirements commonly used during the development of concurrent systems can be, for the most part, covered by a finite set of properties, such as: worst case execution time, worst case traversal time, state duration, schedulability, etc. [1]. As illustrated by Dwyer et. al. [2], property patterns provide a valuable way to handle these requirements. First, they ease the use of formal methods by providing reusable solutions to recurrent specification and verification problems. This is especially true for novice users. Then, they help decomposing complex properties into a set of simpler ones, with a lower complexity, and thus may help decrease the verification cost.

In this paper, we define a new set of property patterns, supporting the definition of timing constraints, that can be easily composed. Each combination of pattern can be translated into a verification observer; therefore providing a way to automatically prove a requirement using a more standard model-checker.

Property patterns are an established concept. In their seminal work, Dwyer et. al. [2] performed a large-scale study of specifications containing over 500 temporal requirements. They noticed that over $90 \%$ of them could be classified under one of the short list of patterns that they had identified. They initially proposed only "qualitative" temporal properties, meaning that

978-1-5386-1925-4/17/\$31.00 (C)2017 European Union they focused on logical time and could not use quantitative concepts, such as time interval or durations.

More recent works [3], [4], [5] have proposed an extension of Dwyer's patterns with real-time constraints. For instance, Konrad et al. [3] mapped quantitative time property patterns into three real-time temporal logics: MTL [6], TCTL [7], and RTGIL [8], thus delegating the verification problem to modelcheckers able to handle these timed logic. They also defined pattern templates to ease reuse. These works were mostly oriented towards providing more expressiveness to users, but did not really address the verification cost associated to each new pattern. Indeed, model checking for MTL, TCTL or RTGIL is expensive in practice and is only available on a very limited number of model-checkers, most of them not maintained anymore. An observer-based approach can help in solving this problem, since it can reduce the initial model checking problem to a much simpler one, like for example a reachability property.

Abid et al. [5] proposed an observer-based verification framework for real-time properties based on Dwyer's work. From a requirement engineering point of view, their patterns are not atomic, meaning that they could be further decomposed into more essential components. Also, they cannot be nested or composed together, but can only be composed through classical disjunction or conjunction of properties. We can illustrate this limitation with the case of the "end-to-end" realtime property (Exist $A$ After $B$ Within I), where $A$ and $B$ are events and $I$ is a given time interval $\left[T_{\min }, T_{\max }\right]$. This pattern means that the first occurrence of $B$ (if it exists) is necessarily followed by an occurrence of $A$ in a delay that is within $I$. In our setting, we can decompose this property into four more basic elements that can be freely composed: (Exist A), (Exist $B),\left(T_{A B} \geq T_{\min }\right)$ and $\left(T_{A B} \leq T_{\max }\right)$, where $T_{A B}$ stands for the possible time interval between the first occurrences of $A$ and $B$. The definition of a more basic library of patterns simplifies the definition of new patterns and their semantics. It also simplifies the verification problem. In [9], Castillos et al. defined a set of compositional automata-based semantics for property patterns, and proposed a composition operation in such a way that the property semantics is defined by composing the automata. This work is limited to "qualitative" temporal properties based on Dewyer's patterns, but does not yet address the "quantitative" ones. 
Our contributions are twofold: (1) the definition of a set of atomic pattern combinators for the formal expression of real-time requirements; and (2) the definition of a set of elementary observers in order to check our real-time patterns. In this work, we use Time Petri Nets (TPN), and an extension of TPN with data called TTS (Time Transition System), as the verification format. We also rely on the model-checking toolbox TINA [10] for the verification part. We define a set of event-based observers at TPN level (12 observers), a set of state-based observers at TTS level (4 observers). Our observers take advantage of the highest possible level of abstraction provided by TINA (marking abstraction in our case) to reduce the size of the abstract state space that needs to be explored during verification. The results of this work has been applied to our UML-MARTE real-time verification framework [11], [12], which provides a toolchain including property-specific model translation [13], property-specific state space reduction [14], probabilistic failure analysis [15], etc.

The paper is organised as follows: Sect. II gives some technical background on model-checking TPN; Sect. III introduces the catalog of real-time property patterns while Sect. IV explains the design of observers; Sect. V illustrates the definition of pattern modifiers and the set of observers; Sect. VI presents the specification and verification on an example of real-time property; Sect. VII gives some concluding remarks.

\section{TECHNICAL BACKGROUND}

Time Petri nets (TPN) [16] are an extension of Petri nets with timing constraints on the transitions. We use an example to explain its syntax and semantics.

The TPN in Fig. 1 models the concurrent execution of a process with two tasks scheduled in parallel. Each transition in a TPN is decorated with a (static) time interval that constraints its firing time. In this net, place $P_{\text {init }}$ is initially marked with one token. Hence transition fork is enabled and should fire immediately (with a delay included in the interval $[0,0]$ ). Upon firing, transitions task 1 and task 2 start at the same date. Intuitively, each transition is associated with a local clock that starts once it is enabled; then the transition can fire when the clock value is in the time interval associated with the transition. For instance, due to their time constraints, Task1_ends always fire before Task2_ends. Once the two tasks finish (there are two tokens in place $P_{\text {join }}$ ), the system can either exit or restart the whole execution.

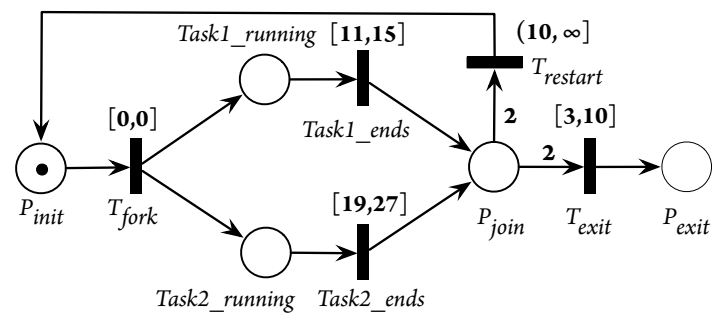

Figure 1. Time Petri Net Example
Time Petri nets can be composed like ordinary Petri net, by combining transitions with a common label. This will be used to add an observer to an existing model. Finally, TTS extend the semantics of TPN by allowing guards on shared data variables in transitions and expressions that are evaluated when a transition fires.

Time Petri Nets provide a formal framework to capture the real-time behavior of concurrent real-time system. There exists several analysis tools, such as TINA or Romeo. Our work relies on TINA (the time Petri net analyzer), a toolbox for the edition and analysis of Petri nets. TINA also supports nets with inhibitor and read arcs, priorities, stopwatches, and its extension TTS. The TINA toolset includes tools for the exploration of reachability graphs (tina and sift) that support a large choice of state abstractions; model-checkers for LTL (selt); for CTL and an existential fragment of $\mu$-calculus (muse); etc.

Next, we define some core concepts used in the specification of patterns: occurrence, predicate, scope, events and states. A pattern describes constraints on three main kind of elements. What is the object of the constraint (predicate)? When should it happen (scope)? And how does it compare with the other events (occurrence)? The occurrence of a predicate could be specified as existence, absence, always (exist), or exist with a bounded number of occurrences. For instance, in our initial example of pattern given in Sect. I, (Exist A After B Within I), the occurrence is Exist, the predicate is the event $A$, and the scope modifier is (After B Within I).

In this context, an event is an instantaneous and atomic occurrence of an action at a point in time. Our patterns can also deal with states, that is identifiers that designate when some given invariant conditions hold. States can change as a result of an event, and we will often talk about the "enter event" of a state. In the remainder of this text, and to avoid ambiguity, a scope modifier will always be applied to an event, while we allow both states and events in a predicate. In particular, if $A$ is a state then the scope After A will apply to the corresponding enter event of $A$.

\section{Catalog of Real-Time Property Patterns}

A real-time property is composed of two main components: a pattern and a scope. Roughly speaking, when considering the state graph of the system, the scope operator is used to select a subset of the reachable states. These (state) candidates are then qualified by the given pattern. Patterns comprises the system devised by Dwyer, that is based on eight patterns (Absence, Existence, Bounded Existence, Precedence, Response, Chain Precedence and Chain Response) and five scope modifiers (Global, Before, After, Between and After-Until). Konrad et al. extended this system to also include quantitative requirements by introducing five quantitative modifiers (Minimum Duration, Maximum Duration, Bounded Recurrence, Bounded Response and Bounded Invariance). We add to this set a new scope modifier, Periodically, for the specification of periodic events related requirements. We also add three real-time suffixes, At least and At most for the specification of bounded time on state 
related predicates, and the suffix Within for the specification of time intervals. The addition of these new modifiers allows us to express more elaborate patterns, such as (At least $d$ Before $E$ ) or (Absent A Precedes B Periodically); etc.

In order to cross the boundary between property specification and verification and reduce the verification cost, we define a set of elementary constructs for property patterns. In the proposed pattern system (see Fig. 2), real-time requirements are specified using either atomic or composite patterns. Composite patterns are built using binary operators (or, and, imply). Atomic patterns are composed of three elementary constructs: occurrence modifier, basic predicate and scope modifier. (To avoid any ambiguities we will reserve the use of the term "pattern" to mean "property pattern".) Basic predicates are defined based on state and event modifiers, and scope modifiers are defined based on event modifiers.

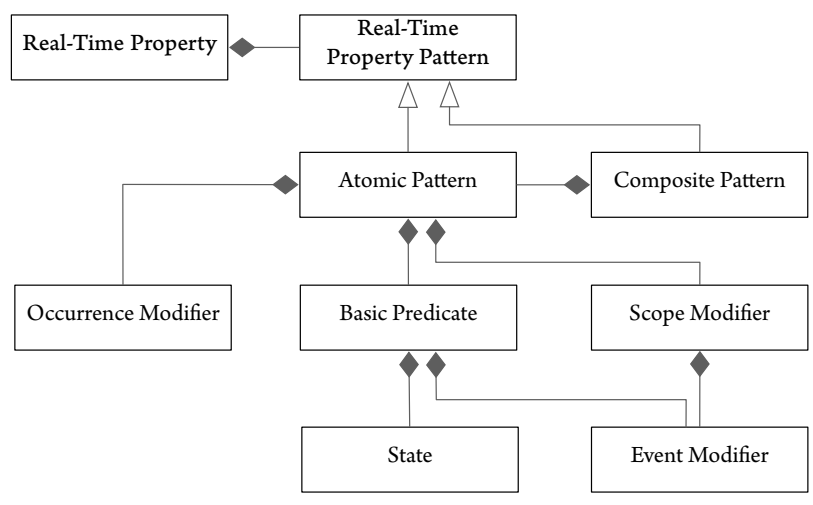

Figure 2. Real-Time Property Specification System

\section{DESIGN PRINCIPLES OF TPN/TTS OBSERVERS}

Before illustrating the set of elementary constructs of property patterns and defining the set of observers, we first explain the design principles of the observers in our model checking approach.

\section{A. Structure of Observer}

A TPN/TTS observer is a sub-net that will be composed with the net capturing the behavior of the system. To assess a property specification based on events, we use a composition described by the diagram of Fig. 3. A TPN/TTS observer is associated to the system through its arcs, joined at the transitions labelled $T_{A}$ and $T_{B}$ in components $\mathrm{A} \& \mathrm{~B}$. On the opposite, a TTS observer for state-based properties is not composed with the system but simply put in parallel (an operation usually referred to as free product). This operation is depicted using arcs with dotted-line in Fig. 3.

The abstract structure of our observers contains a place, namely $P_{\text {tester }}$, which allows properties to be assessed by using accessibility assertions declared within a modal $\mu$-calculus ( $m m c$ ) formula. This $m m c$ formula checks the existence of a specific marking and whether a given set of transitions can be fired. In this context, the most basic $m m c$ formulae that we use are of the form $[T]\left(P_{\text {tester }}=1\right)$ or $\langle T\rangle\left(P_{\text {tester }}=0\right)$, meaning that for all (respectively for at least one) successor state the observer is (respectively is not) in state $P_{\text {tester }}$. All the necessary formulas are checked on-the-fly using the muse model checker.

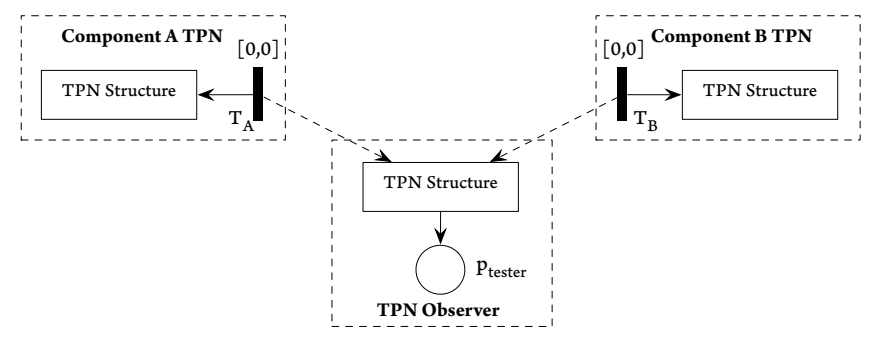

Figure 3. Observer Structure

Compared with the work of Abid et al. [5], we avoid completely the use of LTL model-checking with our observers. Indeed, the use of LTL model-checking requires to use state space abstraction that preserve the set of traces of a language, which can be less effective than abstractions that only preserve state reachability. This has a huge impact on the efficiency of model checking.

\section{B. Soundness of Observer}

Soundness here means that (1) an observer should not impact the system's behavior by introducing extra semantics or removing original semantics; and (2) observers preserve time divergence, meaning that an observer should not be able to stop the evolution of time (introducing some kind of time deadlocks). Indeed, an observer that is synchronized with a place of the system may impact its behavior by adding or removing tokens in an unlawful way. Our approach avoids this problem by interacting with the system only by its transitions. Moreover, the observers work in a "read-only" mode, guaranteed by the design "linked from TPN transitions".

Hence the soundness of observers is achieved using an highlevel property of innocuousness that could be proved formally on each observers, independently from the system. This proof follows the same structure that the one found in the work of Abid et al. [17]. Actually, our group has also conducted experiments on the use of interactive theorem prover to prove this kind of properties [18].

\section{Efficiency of Observer}

We follow three principles to ensure the efficiency of our observers. First, a system with integrated observers should be able to generate state class graphs with a high-level abstraction (i.e. marking abstraction for TINA). This graph should preserve the required semantics of the targeting property. This principle is achieved by forbidding some elements in TPN during the design, such as the priority arcs. We rely on the marking graphs and $m m c$ formulae to transform the quantitative verification problems to reachability problems 
using the muse model checker. Second, the generating state space of a single observer shall be as small as possible. This principle requires us to experiment on different encoding of an observer, in order to select the relatively optimal one. Some experiences are summarized, such that the stopwatch and stopwatch inhibitor arcs are more expensive than the other three types (regular, read, inhibitor arcs). Third, the checking of each property pattern shall be independent to promote parallel computation.

\section{ELEMENTARY OBSERVERS FOR THE VERIFICATION OF PROPERTY PATTERNS}

\section{A. Basic Event Modifiers}

Predicates are specified based on events and states. An event can be an atomic element $E$, or a composite one, called event modifier, e.g. $E^{i}$ for the definition of the $i^{\text {th }}$ occurrence of event $E$. Here is a more complex composite observer: $t$ u.t. (unit of time) after event $E^{i-k}$. It contains three basic event modifiers: (1) $E^{i}$ (the $i^{\text {th }}$ occurrence of event $E$ ); (2) $E^{i-k}$ (the event delayed $k$ times from the current event $E^{i}$ ); and (3) $E^{i-k}+t$ (the event delayed $t$ u.t. from current event $E^{i-k}$ ). We have defined a set of extensible basic event modifiers. As shown in Fig. 4, a generic observer structure for event modifiers are defined, where the transition $E$ is the observable part of the system, and the transition $E^{\prime}$ is the "extensible point" for integrating other event modifiers.

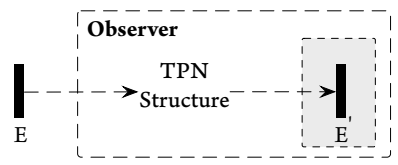

Figure 4. Observer Structure of Event Modifiers

With respect to the above principle, we have defined a set of basic event modifiers.

1) $E^{i}$ : the $i^{\text {th }}$ occurrence of event $E$ : This event modifier, illustrated by Fig. 5, requires that occurrence of $E$ is finite. Note that $E^{1}$ stands for the first occurrence of $E$, while $E$ stands for the event type. If no occurrence is specified, $E$ is regarded as $E^{1}$.

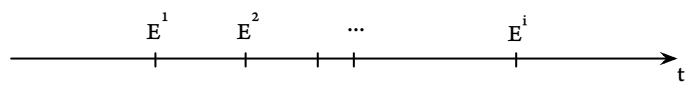

Figure 5. The $i^{\text {th }}$ Occurrence of $E$

Its observer is defined as the TPN in Fig. 6. When $E$ has occurred $i$ times, the place $P_{o c c}$ has $i$ tokens, and the transition $E^{i}$ is enabled. This design ensures that event $E^{i}$ occurs at the same time as the $i^{\text {th }}$ occurrence of event $E$. The place $P_{\text {once }}$ with one token controls the occurrence times of $E^{i}$. It allows $E^{i}$ to occur only once. The number of tokens in $P_{\text {once }}$ can be replaced by another finite value to enable $E^{i}$ several times.

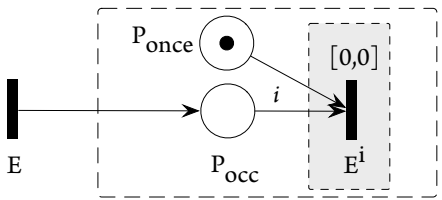

Figure 6. Event Observer: $i^{\text {th }}$ Occurrence of $E$

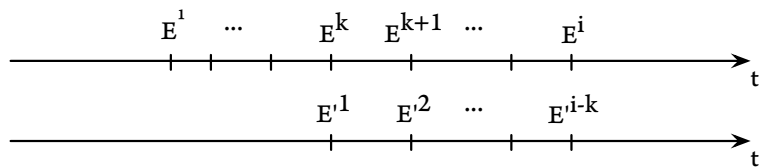

Figure 7. $k$ Times Delay of $E$

2) $E^{-k}: k^{\text {th }}$ delay of $E$ : The event modifier $\mathrm{E}^{-k}$ stands for a delay of $k$ times compared to event $E$, illustrated by Fig. 7 .

The observer of this event modifier is defined in Fig. 8, where the place $P_{o c c}$ stores tokens representing the occurring times of event $E$. Each time $P_{\text {occ }}$ has $k$ tokens, the read arc enables the transition $E^{-k}$, which consumes one token in $P_{o c c}$.

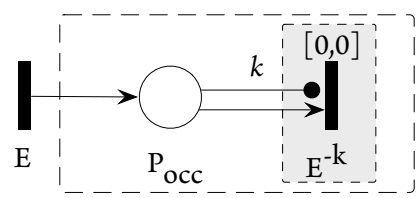

Figure 8. Event Observer: $k$ Times Delay of $E$

3) $E^{\prime k}: k$ times slower sub-occurrence of $E$ : The event modifier $E^{\prime k}$ stands for the sub-occurrence of event $E$, with a frequency $k$ times slower than $E$, illustrated by Fig. 9 .

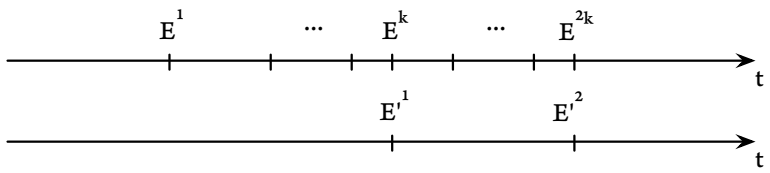

Figure 9. Sub-Occurrence of $E$

Its observer is designed as Fig. 10. When $E$ occurs $k$ times, the place $P_{o c c}$ accumulates $k$ tokens, and the transition $E^{\prime k}$ is fired. Simultaneously, all the $k$ tokens in $P_{o c c}$ are consumed.

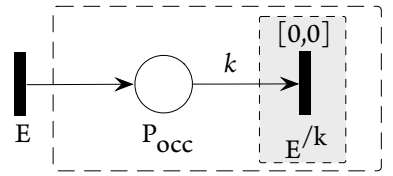

Figure 10. Event Observer: $k$ times slower sub-occurrence of $E$ 
4) I+t: time t elapsed since system initialization: This event modifier $I+t$ stands for the absolute time instant measured from the initial time of the system, illustrated by Fig. 11. It is used to assess properties such as worst/best case execution time.

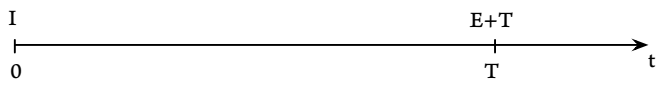

Figure 11. T Time Units after System Initialization

Its observer is designed as Fig. 12, which is composed of two parts: (1) the place $P_{\text {Init }}$ representing the initialization of the system; (2) the transition $E^{\prime}$ representing that $t$ u.t. has elapsed.

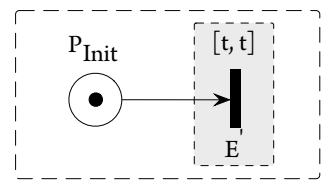

Figure 12. Event Observer: Time Elapsed since System Initialization

5) $E+t$ : time $t$ elapsed since the occurrence of event $E$ : The event modifier $E+t$ stands for the moment when $t$ u.t. has elapsed since the occurrence of $E$, illustrated by Fig. 13 . When using this modifier, the occurring times of $E$ should be finite. This event modifier is used to specify the scope within and the predicates such as at least/at most. For example, the property After $E$ Within I $\left(\left[t_{\min }, t_{\max }\right]\right)$ is specified as After $E+t_{\min }$ and Before $E+t_{\max }$.

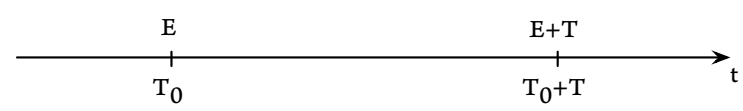

Figure 13. T Time Units after $E$

Its observer is designed as Fig. 14.

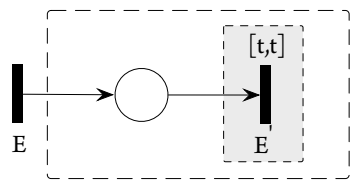

Figure 14. Event Observer: Time Elapsed since $E$

6) $S^{S} \& S^{E}$ : entering and exiting events of a State $S$ : The TTS observer for the entering and exiting events of a state is designed as Fig. 15, where the transitions $S^{S} / S^{E}$ represent the entering/exiting events of the state $S$. When a system enters the state $S$, the assertion $S$ in $\operatorname{PrE}(S)$ is true, which is the guard to enable the transition $S_{S}$ and thus transit the token in the place $P_{S}$ to the place $P_{E}$. Similarly, when the system exits state $S$, the assertion $\neg S$ in $\operatorname{PRE}(\neg S)$ is true, which transits the token in the place $P_{E}$ to the place $P_{S}$.

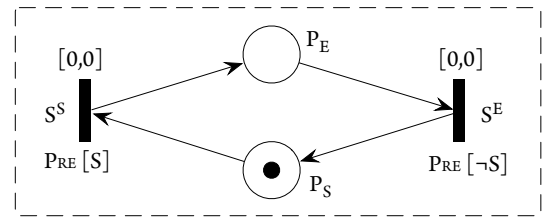

Figure 15. Event Observer: Entering and Exiting Events of S

\section{B. Basic Predicates}

The specification of basic predicates relies on events and states, where an event can be a single event modifiers or a composition of several event modifiers. We have defined a set of basic predicates used by our property patterns. The generic TPN structure of predicate observers is defined as Fig. 16, where the transition $E_{M}$ is an event, and the predicate is assessed using the observer and a set of $m m c$ assertions.

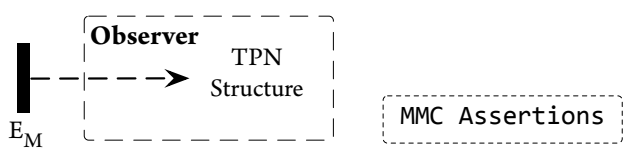

Figure 16. Predicate Observer Pattern

1) $O\left(E^{i}\right)=$ true for the occurrence of event $E^{i}$ : In Fig. 17, the place $P_{o c c}$ linked from transition $E_{M}$ is used to observe the occurrence times of event $E_{M}$. Once the transition $E_{M}$ has fired $i$ times, the token in $P_{o c c}$ is observed, which is assessed using the $m m c$ assertion $P_{o c c} \geq i$. Note that TINA takes $P_{o c c}$ as the number of tokens in the place $P_{o c c}$.

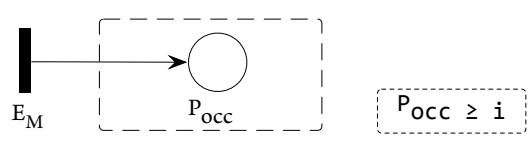

Figure 17. Predicate Observer: Occurrence of $E^{i}$

2) isFinite $(E)=$ True for the bounded occurrence of $E$ : This predicate is used to assess whether the occurrence of an event is finite. In Fig. 18, the place $P_{o c c}$ accumulates the occurrence times of event $E_{M}$. If the transition $T_{\text {Overflow }}$ is not fired, no overflow is detected, as $E_{M}$ does not exceed the occurring bound $O c c_{\max }$, i.e. a predefined threshold value which is usually determined by the estimation on system's behavior.

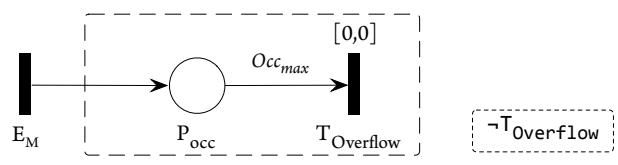

Figure 18. Predicate Observer: Occurrence of $E$ is bounded 
3) $\operatorname{Freq}\left(E_{A}\right) \cdot N_{A}=\operatorname{Freq}\left(E_{B}\right) \cdot N_{B}$ for equivalent occurrence of $E_{A}$ and $E_{B}$ : This predicate is used to identify equivalent occurrences between two periodic events with different (or equal) frequencies. Suppose two periodic events $E_{A}$ and $E_{B}$ exhibit respectively occurrence frequency $F_{A}$ and $F_{B}$. There exists minimal coefficients $N_{A}$ and $N_{B}\left(N_{A}, N_{B} \in \mathbb{Z}^{+}\right)$that makes $F_{A} \cdot N_{A}=F_{B} \cdot N_{B} \cdot N_{A}$ and $N_{B}$ can be computed using the Least Common Multiple (lcm) and the Greatest Common Divisor (gcd).

$$
\begin{aligned}
N_{A} & =\frac{\operatorname{lcm}\left(F_{A}, F_{B}\right)}{\operatorname{gcd}\left(\operatorname{lcm}\left(F_{A}, F_{B}\right), F_{A}\right)} \\
N_{b} & =\frac{\operatorname{lcm}\left(F_{A}, F_{B}\right)}{\operatorname{gcd}\left(\operatorname{lcm}\left(F_{A}, F_{B}\right), F_{b}\right)}
\end{aligned}
$$

A real-time property may require to limit the time difference between two periodic events. If these two events exhibit the same frequency, $N_{A}$ equals to $N_{B}$. Otherwise, $N_{A}$ and $N_{B}$ should be introduced to identify the corresponding occurrence between $E_{A}$ and $E_{B}$.

In Fig. 19, places Tester $_{A} /$ Tester $_{B}$ counts the occurring times of events $E_{A} / E_{B}$. The transition Diff may consume tokens in Tester $_{A} /$ Tester $_{B}$ if tokens in Tester $_{A}$ are superior or equal to $N_{A}$ and tokens in Tester $_{B}$ are superior or equal to $N_{B}$. Once Tester $_{A}$ contains $N_{A}+1$ tokens, $E_{A}$ executes at least one time faster than $E_{B}$. This exception will be detected using the Overflow transitions. The checking assertion is: $\neg\left(\right.$ Overflow $_{A} \vee$ Overflow $\left._{B}\right)$.



Figure 19. Predicate Observer: Same Frequency between $E_{A}$ and $E_{B}$

4) $T\left(E_{A}, E_{B}\right)>t$ for minimal Time Interval between Events: This observer is used to check that the time interval between the equivalent occurrences of $E_{A}$ and $E_{B}$ is at least $t . E_{A}$ and $E_{B}$ can be periodic or aperiodic. Its semantics is equivalent to $\mathrm{T}\left(E_{A}\right)-\mathrm{T}\left(E_{B}\right)>t$. Its observer is similar to that of equivalent occurrence between events, except that a transition $T_{\text {Delay }}$ is added, representing the time delay for event $E_{A}$. We use the following $m m c$ assertion to assess this predicate. When $E_{A}$ and $E_{B}$ are aperiodic, $N_{A}=N_{B}=1$.

$$
\neg\left(\text { Overflow }_{A} \vee \text { Overflow }_{B}\right) \wedge \neg\left(\left(\text { Tester }_{B}=N_{B}\right) \wedge\left(\text { Tester }_{A}<N_{A}\right)\right) .
$$

5) $T\left(E_{A}, E_{B}\right)<t$ for maximum time interval between Events: This observer is used to check the time interval between the equivalent occurrences of $E_{A}$ and $E_{B}$ is at most $t$. Its semantics equals to $\mathrm{T}\left(E_{A}\right)-\mathrm{T}\left(E_{B}\right)<t$. Its observer is designed as Fig. 21. If the assertion $\neg\left(\right.$ Overflow $_{A} \vee$ Overflow $\left._{B}\right)$ is true, then $\operatorname{IT}\left(E_{A}\right)$ - $\mathrm{T}\left(E_{B}\right) \mid<t$ is valid. When $E_{A}$ and $E_{B}$ are aperiodic, $N_{A}=$ $N_{B}=1$.

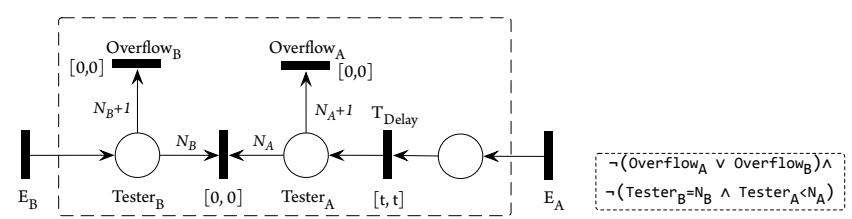

Figure 20. Predicate Observer: Minimum Time Interval between $E_{A}$ and $E_{B}$

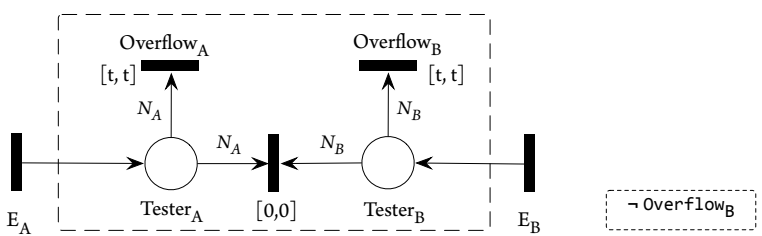

Figure 21. Predicate Observer: Maximum Time Interval between $E_{A}$ and $E_{B}$

6) $D(S) \geq t \& D(S)<t$ for minimal/maximal time duration of a state $S$ : An efficient observer design for the predicate of state duration is to use the PRE function of TTS. In Fig. 22, the transition with constraint $[\mathrm{t}, \mathrm{t}]$ is enabled when state $S$ holds at least/at most $t$ u.t.. The transition with constraint $[0,0]$ will fire when state $S$ does not hold any more. This transition is used to clear the marking in the place Tester, as state $S$ may hold several times in the whole system's execution. The $m m c$ assertions are respectively: $S \wedge($ Tester $=1)$ and $S \wedge($ Tester $=0)$.

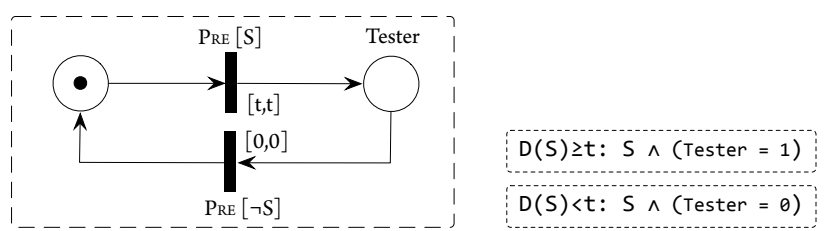

Figure 22. Predicate Observer: Time Duration of State

\section{Basic Scope Modifiers}

Basic scope modifiers include Global, Before $E^{i}$, After $E^{i}$, and Between $E_{A}$ and $E_{B}$. Others are compositions of the basic ones.

1) Global: Global scope modifier does not need an observer in TPN. It requires all states of the system, denoted as $\mathcal{A}$.

2) Before $E^{i} \&$ After $E^{i}$ : The observers for this pair of scope modifiers are designed as the same TPN model, as shown in Fig. 23, but depend on different logic formulae. The place Tester counts the occurring times of event $E$. We use Tester $<i$ ( $E^{i}$ has not yet occurred) to check Before $E^{i}$ and use Tester $\geq i$ ( $E^{i}$ has occurred) to check After $E^{i}$. Note that this scope requires isfinite $(E)$ to be true.

3) Between $E_{A}$ and $E_{B}$ : Between $E_{A}$ and $E_{B}$ means between the equivalent occurrences of $E_{A}$ and $E_{B}$. If both $E_{A}$ and $E_{B}$ are periodic events, their occurrence frequencies must be equal. 


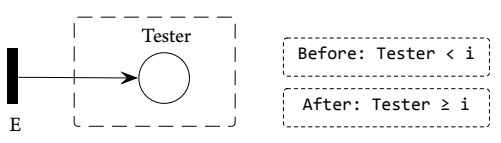

Figure 23. Scope Observer: Before E \& After E

If $E_{A}$ and $E_{B}$ occur only once, by default their frequencies are equal. Its observer is designed as shown in Fig. 24, where the places Tester $_{A}$ and Tester $_{B}$ count the difference of the occurring times between $E_{A}$ and $E_{B}$, and the formula $\left(\right.$ Tester $\left._{A}=1\right) \wedge\left(\right.$ Tester $\left._{B}=0\right)$ is used for the assessment.

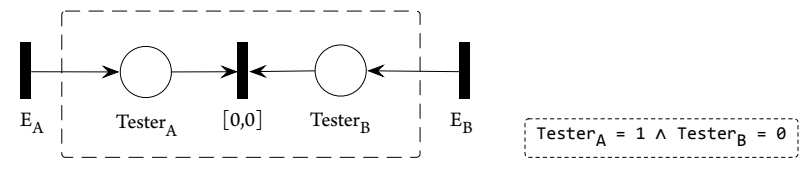

Figure 24. Scope Observer: Between two Events

4) After $E_{A}$ Until $E_{B}$ : This scope modifier can be represented by the above ones:

- When $E_{B}$ occurs after $E_{A}$, it is equivalent to (Exist $E_{B}$ After $E_{A}$ ) and (Between $E_{A}$ and $E_{B}$ );

- When $E_{B}$ does not occurs after $E_{A}$, it is equivalent to (Absent $E_{B}$ After $\left.E_{A}\right)$ and (After $\left.E_{A}\right)$.

\section{Occurrence Modifiers}

Occurrence modifiers are used to specify the occurrence times of given event/state modifiers within some scope. They are classified as Exist, Absent, and Always, and are used together with predicates and scopes. The use of observers for occurrence modifiers is not mandatory. Assume that in the state graph, $N(P)$ is the number of states that match the predicate $P, N(S)$ is the number of states that match the scope $S$, and $N(P \wedge S)$ is the number of states that match both the predicate and the scope. With respect to the semantics of Exist, Absent, Always, the assessments depend on the following assertions:

- Exist $P$ in $S$ means that $P$ must occur within $S$ :

$$
\begin{cases}N(P \wedge S) \geq 1 & \text { if } \mathrm{N}(\mathrm{S})>0 \\ \text { True } & \text { if } \mathrm{N}(\mathrm{S})=0 .\end{cases}
$$

- Absent $P$ in $S$ means that $P$ must not occur in $S$ :

$$
N(P \wedge S)=0
$$

- Always $P$ in $S$ means that $P$ occur through the whole $S$ :

$$
N(P \wedge S)=N(S)
$$

Note that when $N(S)=0$, the scope is false, then the predicate for any occurrence modifier (Exist, Absent, Always) is always true. The assertions for Absent and Always satisfies this fact by default. The assertion "True, if $\mathrm{N}(\mathrm{S})=0$ " for Exist is also defined for this purpose.

\section{EXAmple of Real-Time Property Verification}

We illustrate the proposed specification and verification approach using a simple example (see Ex. 1).

Example 1: As shown in Fig. 25, two concurrent processes are modeled in TPN. Both execute only once. The target property $\mathcal{P}$ is Always $E_{A}$ After $E_{B}$ Within $[1,2]$.
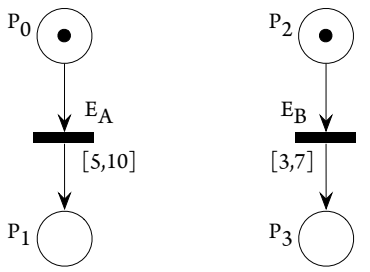

Figure 25. Observer-based Verification Example

On the basis of our specification patterns, $\mathcal{P}$ is composed by (1) the occurrence modifier Always, (2) the predicate $E_{A}$ occurs, and (3) the scope After $E_{B}$ Within [1,2], which is equivalent to Between $E_{B}+1$ and $E_{B}+2$.

Accordingly, observers for assessing $\mathcal{P}$ are generated using the set of observers defined in our approach, as shown in Fig. 26. The predicate $E_{A}$ occurs is observed by using an atomic event-based observer $\mathbf{o b s}_{\mathbf{4}}$. The scope Between $E_{B}+1$ and $E_{B}+2$ is observed by using a composite observer with three parts: (i) $\mathbf{o b s}_{\mathbf{1}}$ for event modifier $E_{B}+1$, (ii) $\mathbf{o b s}_{\mathbf{2}}$ for event modifier $E_{B}+2$, and (iii) $\mathbf{o b s}_{3}$ for scope modifier Between $E_{B}+1$ and $E_{B}+2$.

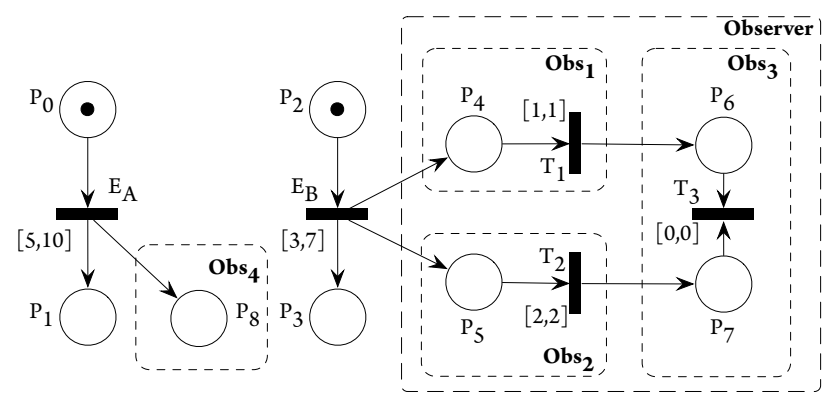

Figure 26. TPN Observers for the Example

Based on the TPN system with additional observers, a state graph of marking abstraction with 10 states and 13 transitions is generated, shown in Fig. 27. Relying on the muse model checker, $\mathcal{P}$ is assessed using the following $m m c$ formulae:

- Assertion $P$ for the predicate $E_{A}$ occurs: $P_{8}$.

- Assertion $S$ for the scope Between $E_{B}+1$ and $E_{B}+2$ : $P_{6} \wedge \neg P_{7}$.

- Assertion $O$ for the occurrence modifier Always: if $N(P \wedge S)=N(S)$, then $P$ is satisfied.

For the assertion $O$, only one state $\left(S_{5}\right)$ satisfies $P \wedge S$, thus $N(P \wedge S)=1$; while two states $\left(S_{4}\right.$ and $\left.S_{5}\right)$ satisfy $S$, thus $N(S)=2$. As $N(P \wedge S) \neq N(S)$, property $\mathcal{P}$ fails. 


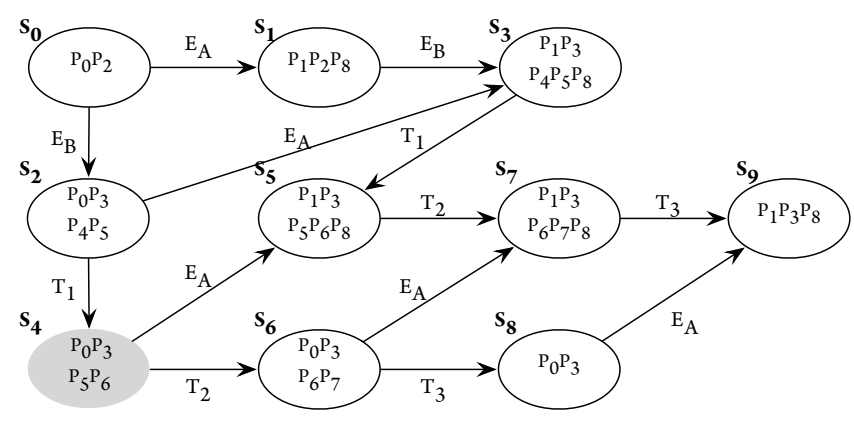

Figure 27. Reachability Graph of Verification Example

$N(P \wedge S)<N(S)$ implies that the states satisfying $\neg P \wedge S$ $\left(\neg P_{1} \wedge P_{6} \wedge \neg P_{7}\right)$ are violating ones. By checking $\neg P_{1} \wedge P_{6} \wedge$ $\neg P_{7}$, we find the violating state in $k t z$, which is the state $S_{4}$ with marking $P_{0} P_{3} P_{5} P_{6}$.

\section{CONCLUSION}

Dwyer's and Konrad's pattern systems target expressiveness of real-time requirements for the end-users, and leave the verification related issues to the users. Accordingly, these patterns do not guarantee the efficiency of verification. We have defined a set of atomic constructs in our property pattern system and defined the associated set of elementary observers. End-user real-time requirements are expressed as compositions of these patterns based on a predefined meta-model and a set of mapping rules.

Compared to previous works from one of the author [5], our proposal allows for the automatic generation of composite observers for a composite property. It has also a positive outcome on the verification cost. Our pattern verification framework has been integrated into our UML-MARTE realtime verification framework [11], [12]. This extends our available tooling for UML-MARTE with a process for formal requirement verification and elicitation during the early design phases.

\section{ACKNOWLEDGMENT}

This work was funded by the FUI Project P and ITEA2 Project OPEES projects.

\section{REFERENCES}

[1] H. Kopetz, Real-time systems: design principles for distributed embedded applications. Springer, 2011.

[2] M. B. Dwyer, G. S. Avrunin, and J. C. Corbett, "Patterns in property specifications for finite-state verification," in Proceedings of the 21st International Conference on Software Engineering, ser. ICSE '99. ACM, 1999, pp. 411-420.

[3] S. Konrad and B. H. Cheng, "Real-time specification patterns," in Proceedings of the 27th international conference on Software engineering. ACM, 2005, pp. 372-381.

[4] V. Gruhn and R. Laue, "Patterns for timed property specifications," Electronic Notes in Theoretical Computer Science, vol. 153, no. 2, pp. $117-133,2006$.

[5] N. Abid, S. Dal Zilio, and D. Le Botlan, "Real-time specification patterns and tools," in Formal Methods for Industrial Critical Systems. Springer, 2012, pp. 1-15.

[6] R. Koymans, "Specifying real-time properties with metric temporal logic," Real-time systems, vol. 2, no. 4, pp. 255-299, 1990.

[7] R. Alur, "Techniques for automatic verification of real-time systems," Ph.D. dissertation, stanford university, 1991.

[8] L. E. Moser, Y. Ramakrishna, G. Kutty, P. M. Melliar-Smith, and L. K. Dillon, "A graphical environment for the design of concurrent real-time systems," ACM Transactions on Software Engineering and Methodology (TOSEM), vol. 6, no. 1, pp. 31-79, 1997.

[9] K. C. Castillos, F. Dadeau, J. Julliand, B. Kanso, and S. Taha, "A compositional automata-based semantics for property patterns," in International Conference on Integrated Formal Methods. Springer, 2013, pp. 316330.

[10] B. Berthomieu, P.-O. Ribet, and F. Vernadat, "The tool TINAconstruction of abstract state spaces for Petri nets and Time Petri nets," International Journal of Production Research, vol. 42, no. 14, 2004.

[11] N. Ge and M. Pantel, "Time properties verification framework for umlmarte safety critical real-time systems," in European Conference on Modelling Foundations and Applications. Springer, 2012, pp. 352367.

[12] N. Ge, M. Pantel, and X. Crégut, "A UML-MARTE temporal property verification tool based on model checking," in International Conference on Embedded Real Time Software and Systems (ERTS), 2014.

[13] N. Ge, M. Pantel, and X. Crégut, "Time properties dedicated transformation from UML-MARTE activity to time transition system," $A C M$ SIGSOFT Software Engineering Notes, vol. 37, no. 4, pp. 1-8, 2012.

[14] N. Ge and M. Pantel, "Real-time property specific reduction for Time Petri net," in International Workshop on Petri Nets and Software Engineering (PNSE@PetriNets), 2014,pp. 165-179.

[15] N. Ge, M. Pantel, and X. Crégut, "Automated failure analysis in model checking based on data mining," in International Conference on Model and Data Engineering. Springer, 2014, pp. 13-28.

[16] P. Merlin and D. Farber, "Recoverability of communication protocolsimplications of a theoretical study," Communications, IEEE Transactions on, vol. 24, no. 9, pp. 1036 - 1043, 1976

[17] N. Abid, S. Dal Zilio, and D. Le Botlan, "A formal framework to specify and verify real-time properties on critical systems," IJCCBS, vol. 5, no. $1 / 2,2014$.

[18] M. Garnacho, J. Bodeveix, and M. Filali-Amine, "A mechanized semantic framework for real-time systems," in Formal Modeling and Analysis of Timed Systems FORMATS, 2013. 